\title{
Adaptive Gain Control Method of a Phase-Locked Loop for GNSS Carrier Signal Tracking
}

\author{
Zhibin Luo $\mathbb{D}^{\text {, Jicheng Ding }} \mathbb{D}$, and Lin Zhao \\ College of Automation, Harbin Engineering University, Harbin, China \\ Correspondence should be addressed to Jicheng Ding; aaron.heu@163.com
}

Received 7 October 2017; Accepted 11 December 2017; Published 4 March 2018

Academic Editor: N. Nasimuddin

Copyright (c) 2018 Zhibin Luo et al. This is an open access article distributed under the Creative Commons Attribution License, which permits unrestricted use, distribution, and reproduction in any medium, provided the original work is properly cited.

\begin{abstract}
The global navigation satellite system (GNSS) has been widely used in both military and civil fields. This study focuses on enhancing the carrier tracking ability of the phase-locked loop (PLL) in GNSS receivers for high-dynamic application. The PLL is a very popular and practical approach for tracking the GNSS carrier signal which propagates in the form of electromagnetic wave. However, a PLL with constant coefficient would be suboptimal. Adaptive loop noise bandwidth techniques proposed by previous researches can improve PLL tracking behavior to some extent. This paper presents a novel PLL with an adaptive loop gain control filter (AGCF-PLL) that can provide an alternative. The mathematical model based on second- and third-order PLL was derived. The error characteristics of the AGCF-PLL were also derived and analyzed under different signal conditions, which mainly refers to the different combinations of carrier phase dynamic and signal strength. Based on error characteristic curves, the optimal loop gain control method has been achieved to minimize tracking error. Finally, the completely adaptive loop gain control algorithm was designed. Comparable test results and analysis using the new method, conventional PLL, FLL-assisted PLL, and FAB-LL demonstrate that the AGCF-PLL has stronger adaptability to high target movement dynamic.
\end{abstract}

\section{Introduction}

The global navigation satellite system (GNSS) is a system that uses satellites to provide autonomous positioning, which has been widely used in both military and civil fields. It allows electronic receivers that are of small volume to determine their navigation parameters (i.e., position, velocity, and time (PVT)) with high accuracy using signals transmitted by satellites anytime and anywhere around the globe. The GNSS carrier signal transmitted by satellites propagates in the form of electromagnetic wave with binary navigation data and pseudorandom noise (PRN) code modulated on it. After being transmitted by GNSS satellites, the GNSS signal would propagate through the atmosphere, subsequently being received by an antenna of the GNSS receiver near the ground and further processed by the receiver itself. So far, there exist four GNSS in the world. They are the Chinese Beidou System (BDS), the
American Global Positioning System (GPS), the European Union's Galileo Navigation Satellite System, and the Russian GLONASS.

In the process of GNSS signal propagation, the frequency of the carrier signal received by the GNSS receiver differs from the frequency of the carrier transmitted by a satellite. This phenomenon is referred to as the Doppler effect which is caused by relative motion between the GNSS satellites and GNSS receiver. Fortunately, it is the Doppler effect that provides us a method to measure the relative velocity between the receiver and satellites, which is the precondition of receiver velocity determination. That is, the technique to compute the velocity of a user is based on the received carrier Doppler frequency or phase measurements between several satellites and the receiver. Specifically, the receiver has to synchronize its own local carrier with the incoming signals to extract the navigation data and perform the Doppler measurements. Traditionally, carrier synchronization is achieved 
by a carrier tracking loop, which is often implemented as PLL. Apart from PLL, a frequency-locked loop (FLL) and its combination with PLL are also common carrier tracking loop structures to improve receivers' dynamic performance [1]. Besides, vector tracking algorithms and sensor-aided GNSS carrier tracking techniques are very popular in robust receiver design [2]. The carrier tracking loop is designed to track the incoming carrier's phase dynamic or its Doppler frequency caused by the relative motion between the receiver and satellites. The Doppler frequency estimated by the carrier tracking loop mainly contains three terms: the true Doppler frequency, the receiver clock drift, and the noise [2]. The second term could be formulated after obtaining the receiver velocity. However, the noise term, which results from signal propagation and receiver processing, cannot be estimated exactly. Therefore, the carrier tracking loop is also designed to filter the noise in the incoming carrier so that more precise carrier phase or Doppler frequency measurements can be obtained, which will bring about more precise velocity measurements.

Generally, the function of PLL in GNSS receivers is to try to reduce the phase difference between the incoming carrier and local generated carrier. When PLL is in a locking state, the local carrier can be considered a precise replica of the received carrier. Inevitably, the measurements for the incoming carrier phase contain error. The dominant sources of phase error are phase jitter which is mainly caused by thermal noise and dynamic stress error which is caused by relative motion between the receiver and satellites [3]. For PLL, dynamic stress error could be relieved by increasing the PLL loop noise bandwidth whereas phase jitter error can be reduced by decreasing the PLL loop noise bandwidth. That means that improving receiver noise performance would degrade its dynamic performance. Hence, the confliction between PLL dynamic performance and noise performance necessitates some compromises in PLL design, and many methods have been developed to address this typical issue [4-8].

A method to determine appropriate loop noise bandwidth is considering the worst carrier-to-noise power density ratio (CNR) and max relative dynamic (i.e., max relative acceleration for the 2nd-order PLL and max relative jerk for the 3rd-order PLL) [4]. Unfortunately, it will lead to a suboptimal loop for low relative dynamic. A PLL with two sets of loop coefficients working for pull-in mode and tracking mode, respectively, can decrease pull-in time [5]. However, it is hard to improve carrier tracking capability. Adapting loop noise bandwidth depending on the real-time estimations of the relative dynamics and CNR, known as fast adaptive bandwidth lock loops (FAB-LL), is a real-time optimal PLL through minimizing the thermal noise within the total tracking error $[6,9]$. The continuation of FAB-LL has been also proposed in [10]. The second version of FAB-LL is able to automatically and dynamically set loop noise filter parameters of PLL and DLL in order to minimize the total tracking error. However, the noisy and timevarying $\mathrm{CNR}$ and relative dynamic estimations which might cause intense and frequent loop noise bandwidth adjustment limit its application. The PLL does not allow its coefficient to change quickly; otherwise, the loop filter will output impulses resulting in PLL losing lock [6]. To solve this problem, a simple method is to insert a lowpass filter to delay the bandwidth change rate [3].

Besides, PLL behavior can be affected significantly by a loop gain, which is not discussed in detail in previous research. Researches have demonstrated that a reasonable loop gain could promote carrier tracking performance $[11,12]$. By contrast, an adaptive loop gain control method assisted by FLL has been designed for fast convergence from pull-in to tracking mode and decreasing tracking error when PLL has to deal with high-dynamic movement [7]. This method establishes an empirical relation between the loop gain and frequency difference between the received carrier and local carrier. The loop gain could be adaptively adjusted according to the frequency discriminator outputs. However, the use of empirical relation without a mathematical model accurately modeling and analyzing the system leads to a suboptimal PLL. The performance of this kind of method has not been compared with that of FAB-LL. A mathematical model describing the 2nd-order PLL with a loop gain control factor has been derived in [8]. It has been shown that a loop gain can control loop noise bandwidth and characteristic frequency. According to its results, an optimal loop gain can be calculated to minimize the dominating tracking error in different signal conditions. It also shows the possibility that the loop gain could be adjusted adaptively based on CNR and relative dynamic estimations. Furthermore, the 3rd-order PLL can track the phase acceleration input and has advantage over the 2nd-order PLL in tracking carrier phase dynamic. From the comprehensive theoretical research perspective, the modeled relationship should also be analyzed.

The focus of this paper is the development and analysis of an adaptive loop gain control technique for 2nd- and 3rd-order PLL to enhance PLL high-dynamic adaptability. The structure of the paper is shown as follows: In Section 2, the mathematical model of AGCFPLL is derived. A loop gain control factor is inserted into the traditional PLL to adjust the loop gain conveniently. In order to avoid intense adjustment of a loop gain, an inertial element is added to make the loop gain adjustment smoother. In Section 3, the relationship between the behavior of AGCF-PLL and its loop gain is analyzed in detail in time and frequency domains, respectively. In Section 4, an optimal loop gain adjustment strategy is designed to minimize the dominating tracking error according to estimated CNR and relative dynamic between the receiver and satellites. A simple averaging operation can be used to reduce the noise in the relative acceleration estimations and relative jerk estimations. Comparable tests and analysis are conducted between the novel adaptive loop gain control PLL, the conventional PLL, the FLL-assisted PLL, and the FAB-LL, to show the loop dynamic adaptability of the novel algorithms in Section 5. Finally, some concluding remarks are given in Section 6. 


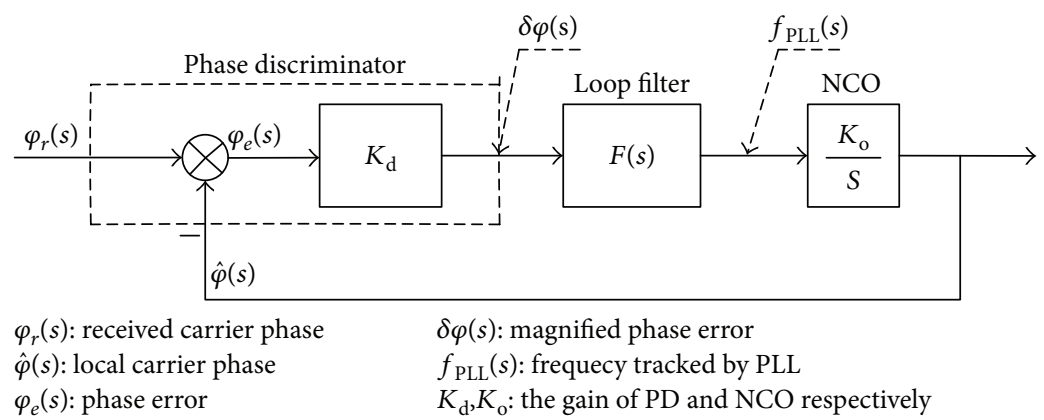

FIGURe 1: Traditional linear PLL model. $\varphi_{r}(s)$ : received carrier phase; $\widehat{\varphi}(s)$ : local carrier phase; $\varphi_{e}(s)$ : phase error; $\delta \varphi(s)$ : magnified phase error; $f_{\mathrm{PLL}}(s)$ : frequency tracked by PLL; $K_{\mathrm{d}}$ : gain of PD; $K_{\mathrm{o}}$ : gain of NCO.

\section{GNSS Carrier Tracking Loop with an Adaptive Loop Gain Control Filter (AGCF-PLL)}

This section reviews the basic concepts of the traditional GNSS carrier tracking loop. The idea to incorporate the loop gain control filter to change the loop gain conveniently and smoothly will build upon the traditional loop structure, and the mathematical model of this new PLL is derived. Then, the digital implementation of this kind of PLL will be discussed. The traditional PLL linear model was discussed in more detail in $[3,5,12-16]$.

2.1. Traditional Linear Model of PLL. The function of PLL within GNSS receivers is to try to generate a local sinusoidal carrier whose phase is the same with the received carrier phase. When PLL is in a locking state, the received carrier phase can be measured by observing the local generated carrier. Meantime, the carrier is wiped off from the received signal. Figure 1 shows the traditional linear PLL model. There are three components: phase discriminator (PD), loop filter (LF), and numerically controlled oscillator (NCO). The phase discriminator calculates the phase difference between the received carrier and local carrier. The phase difference is sent into the loop filter which not only filters the noise but also generates the control signals to NCO. NCO transforms the control signals into local carrier phase adjustment so that the local carrier phase is precisely aligned with the received carrier phase.

The PLL transfer function and its error transfer function can be derived from Figure 1 as

$$
\begin{gathered}
H(s)=\frac{\widehat{\varphi}(s)}{\varphi_{r}(s)}=\frac{K F(s)}{s+K F(s)}, \\
H_{e}(s)=\frac{\varphi_{e}(s)}{\varphi_{r}(s)}=\frac{s}{s+K F(s)},
\end{gathered}
$$

where $F(s)$ is the transfer function of the loop filter and $K$ is the loop gain that is defined as

$$
K=K_{\mathrm{o}} K_{\mathrm{d}}
$$

1st-order LF and 2nd-order LF are often utilized to track certain phase dynamic in a received signal [17]. The 2nd- order PLL with a 1st-order loop filter is able to track phase acceleration input without steady-state error, which is caused by velocity in relative movement. The 3rd-order PLL with a 2nd-order loop filter is able to track phase jerk input caused by acceleration in relative movement without steady-state error. The transfer functions of 2 nd-order and 3 rd-order loops are expressed as follows:

$$
\begin{aligned}
& F_{1}(s)=\frac{1}{K}\left(2 \xi \omega_{n 1}+\frac{\omega_{n 1}^{2}}{s}\right)(1 \text { st-order LF }), \\
& F_{2}(s)=\frac{1}{K}\left(b \omega_{n 2}+\frac{a \omega_{n 2}^{2}}{s}+\frac{\omega_{n 2}^{3}}{s^{2}}\right)(2 \text { nd-order LF }),
\end{aligned}
$$

where $\xi$ is the damping factor of the 2 nd-order loop, $a$ and $b$ are the loop coefficients of the 3rd-order loop, and $\omega_{n 1}$ and $\omega_{n 2}$ are the natural frequencies of the 2nd-order and 3rdorder PLL, respectively.

Substituting (3) into (1), one can rewrite the 2 nd-order PLL system transfer function and error transfer function as

$$
\begin{gathered}
H_{1}(s)=\frac{2 \xi \omega_{n 1} s+\omega_{n 1}^{2}}{s^{2}+2 \xi \omega_{n 1} s+\omega_{n 1}^{2}}, \\
H_{e 1}(s)=\frac{s^{2}}{s^{2}+2 \xi \omega_{n 1} s+\omega_{n 1}^{2}} .
\end{gathered}
$$

Similarly, system transfer function and error transfer function for the 3rd-order PLL are given by

$$
\begin{aligned}
& H_{2}(s)=\frac{b \omega_{n 2} s^{2}+a \omega_{n 2}{ }^{2} s+\omega_{n 2}{ }^{3}}{s^{3}+b \omega_{n 2} s^{2}+a \omega_{n 2}^{2} s+\omega_{n 2}{ }^{3}}, \\
& H_{e 2}(s)=\frac{s^{3}}{s^{3}+b \omega_{n 2} s^{2}+a \omega_{n 2}^{2} s+\omega_{n 2}{ }^{3}} .
\end{aligned}
$$

2.2. Model of AGCF-PLL. In order to control the loop gain conveniently and smoothly according to different relative dynamics and signal strengths, a loop gain control filter can be inserted into the traditional PLL structure, including a gain control factor $\left(K_{\mathrm{c}}\right)$ and inertial element $(I(s))$ as shown 


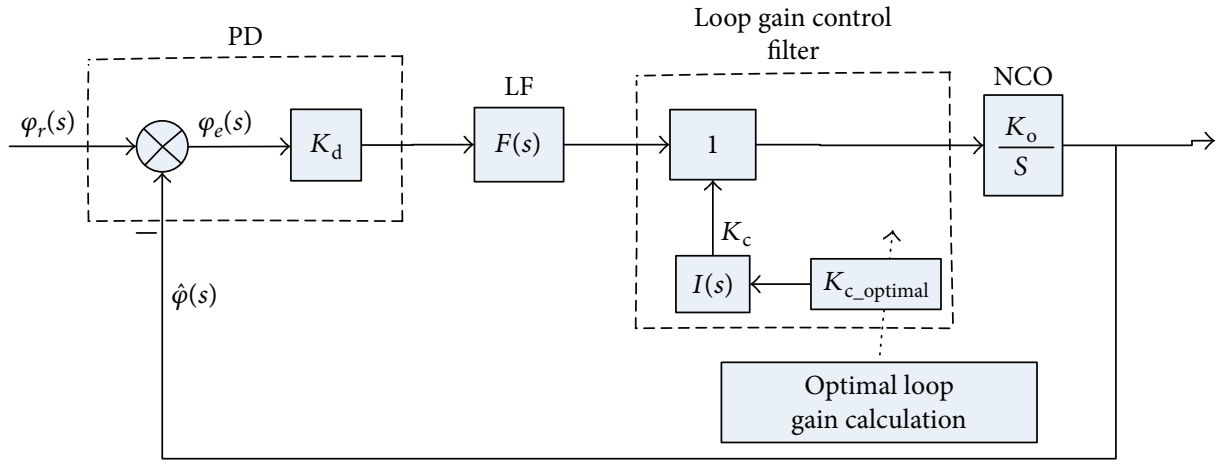

Figure 2: PLL model with a loop gain control filter.

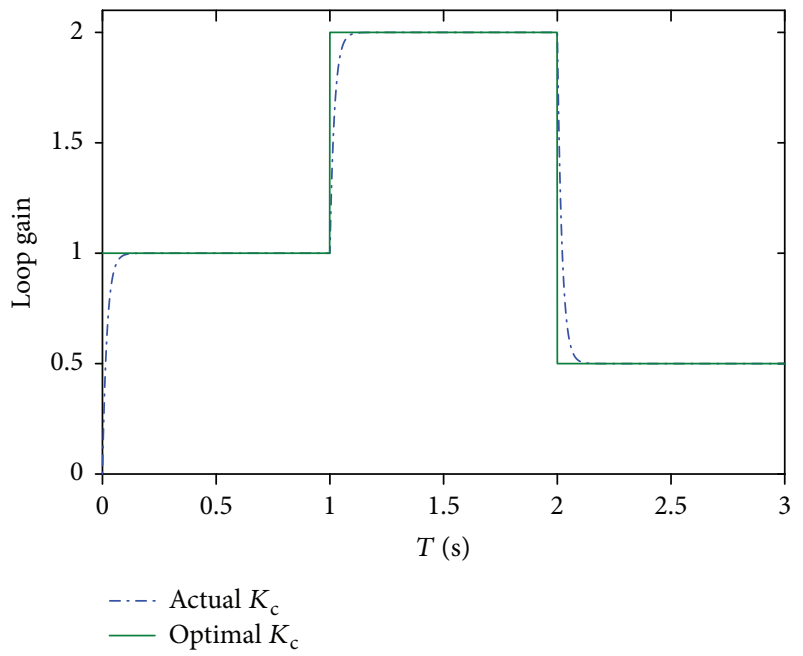

FIgURE 3: The principle of loop gain adjustment.

in Figure 2. Consequently, the actual loop gain could be expressed as

$$
K^{\prime}=K_{\mathrm{c}} K_{\mathrm{o}} K_{\mathrm{d}}
$$

The loop gain could then be adjusted by $K_{c}$. Generally, $K_{\mathrm{o}}$ and $K_{\mathrm{d}}$ are set to one when arc tangent phase discriminator is employed. Thus, $K_{c}$ is the actual loop gain. The transfer function of the loop gain control filter is

$$
K_{\mathrm{c}}(s)=\frac{K_{\mathrm{c} \_ \text {optimal }}}{T s+1},
$$

where $T$ is the time constant of the inertial element in the unit of seconds and $K_{\text {c_optimal }}$ is the optimal loop gain which can minimize the dominating tracking error. The inertial element can delay the change rate of the actual loop gain, as shown in Figure 3. It takes about $3 T$ seconds before the actual loop gain reaches its steady value when the optimal loop gain changes.

Figure 4 shows the distribution rejection ability of the inertial element in loop coefficient adjustment. Here, we create a scene where there are some step changes in loop gain adjustment. There are two options in loop gain adjustment. The loop gain is adjusted directly in option 1, while the loop
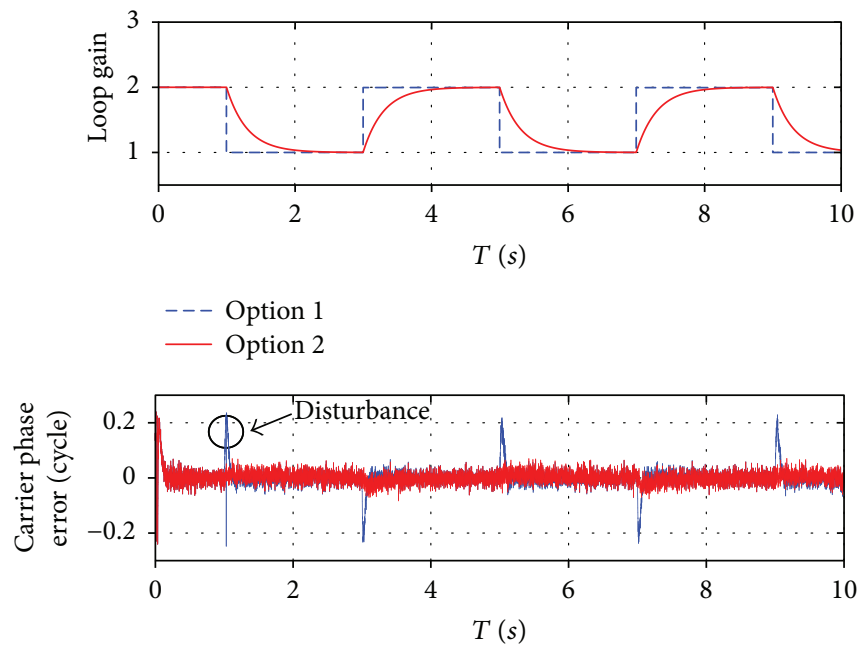

- Option 1

- Option 2

FIgURE 4: The disturbance rejection ability of the inertial element in loop coefficient adjustment.

gain passed the inertial element in option 2 . As shown in the above figure of Figure 4 . The ability of the inertial element rests in the fact that it would delay the change rate of the loop gain. The below figure in Figure 4 shows the carrier phase error discriminator output in two options. The discriminator output represents the carrier phase tracking error and reflects the locking condition of PLL for the carrier phase. It is obvious that the direct loop gain adjustment will introduce disturbance to the tracking loop. It takes some time before the PLL recovers to a locking state from this disturbance. As for option 2, the inertial element adjusts the loop gain in a smooth way so that the disturbance is eliminated. Another function of the inertial element lies in decreasing the noisy optimal $K_{\mathrm{c}}$ calculation, which will be explained in detail in Section 4.

According to Figure 2, the 2nd- and 3rd-order AGCFPLL transfer function and error transfer function can be derived to be their general forms as follows:

$$
H_{3}(s)=\frac{K_{c} K F_{1}(s)}{s+K_{c} K F_{1}(s)}=\frac{2 \xi \omega_{n 1} \cdot K_{c} s+\omega_{n 1}^{2} \cdot K_{c}}{s^{2}+2 \xi \omega_{n 1} \cdot K_{c} s+\omega_{n 1}^{2} \cdot K_{c}},
$$




$$
\begin{aligned}
H_{e 3}(s) & =\frac{s}{s+K_{c} K F(s)}=\frac{s^{2}}{s^{2}+2 \xi \omega_{n 1} \cdot K_{c} s+\omega_{n 1}^{2} \cdot K_{c}}, \\
H_{4}(s) & =\frac{K_{c} K F_{2}(s)}{s+K_{c} K F_{2}(s)} \\
& =\frac{K_{c} \cdot b \omega_{n 2} s^{2}+K_{c} \cdot a \omega_{n 2}^{2} s+K_{c} \cdot \omega_{n 2}{ }^{3}}{s^{3}+K_{c} \cdot b \omega_{n 2} s^{2}+K_{c} \cdot a \omega_{n 2}{ }^{2} s+K_{c} \cdot \omega_{n 2}{ }^{3}}, \\
H_{e 4}(s) & =\frac{s}{s+K_{c} K F_{2}(s)} \\
& =\frac{s^{3}}{s^{3}+K_{c} \cdot b \omega_{n 2} s^{2}+K_{c} \cdot a \omega_{n 2}{ }^{2} s+K_{c} \cdot \omega_{n 2}{ }^{3}} .
\end{aligned}
$$

The inertial element is excluded when deriving these equations because of its delay function. It should be noted that actual $K_{\mathrm{c}}$ would follow optimal $K_{\mathrm{c}}$. Furthermore, the inertial element can be ignored when $K_{c}$ reaches its steady value.

To normalize the AGCF-PLL models for the sake of finding the relationship of loop coefficients between the ordinary PLL and AGCF-PLL, two sets of new loop filter coefficients are introduced here. The first set of new coefficients is used to normalize the 2nd-order AGCF-PLL and defined as follows:

$$
\begin{gathered}
\xi^{\prime}=\sqrt{K_{c}} \xi, \\
\omega_{n 1}^{\prime}=\sqrt{K_{c}} \omega_{n 1} .
\end{gathered}
$$

Substituting (12) and (13) into (8) and (9), one can rewrite the 2nd-order AGCF-PLL transfer function and error transfer function to be their normalized forms as

$$
\begin{gathered}
H_{3}(s)=\frac{2 \xi^{\prime} \omega_{n 1}^{\prime} s+\omega_{n 1}^{\prime 2}}{s^{2}+2 \xi^{\prime} \omega_{n 1}^{\prime}+\omega_{n 1}^{\prime 2}}, \\
H_{e 3}(s)=\frac{s^{2}}{s^{2}+2 \xi^{\prime} \omega_{n 1}^{\prime}+\omega_{n 1}^{\prime 2}} .
\end{gathered}
$$

Obviously, these two equations are similar to the model of the ordinary 2 nd-order PLL, as expressed in (4).

The second set of coefficients is used to normalize the 3rd-order AGCF-PLL and stated as follows:

$$
\begin{aligned}
\omega_{n 2}^{\prime} & =\sqrt[3]{K_{c}} \omega_{n 2}, \\
a^{\prime} & =\sqrt[3]{K_{c}} a \\
b^{\prime} & =K_{c}^{2 / 3} b .
\end{aligned}
$$

Substituting (15) and (16) into (10) and (11), one can rewrite the 3rd-order AGCF-PLL transfer function and error transfer function to be their normalized forms as

$$
\begin{aligned}
& H_{4}(s)=\frac{b \omega_{n 2}^{\prime} s^{2}+a^{\prime} \omega_{n 2}^{\prime 2} s+\omega_{n 2}^{\prime 3}}{s^{3}+b^{\prime} \omega_{n 2}^{\prime} s^{2}+a^{\prime} \omega_{n 2}^{\prime 2} s+\omega_{n 2}^{\prime 3}}, \\
& H_{e 4}(s)=\frac{s^{3}}{s^{3}+b^{\prime} \omega_{n 2}^{\prime} s^{2}+a^{\prime} \omega_{n 2}^{\prime 2} s+\omega_{n 2}^{\prime 3}} .
\end{aligned}
$$

It is obvious that these two equations are similar to the model of the ordinary 3rd-order PLL, as expressed in (5).

By comparing the ordinary PLL model with the normalized AGCF-PLL model, it can be found that they have similar form with different loop coefficients. The change of $K_{\mathrm{c}}$ in AGCF-PLL is equivalent to change loop coefficients in the ordinary PLL. This relation is shown in (12), (13), (15), (16), and (17). It is easy to find out that the AGCF-PLL is equivalent to the ordinary PLL if the loop gain is set to one.

2.3. Digital Implement of AGCF-PLL. In GNSS receivers, the PLL is implemented in the form of a Costas loop which is insensitive to phase transitions due to navigation bits [15]. Figure 5 shows the digital implementation of AGCFPLL including components like mixers and integration and dump operations.

The digital intermediate frequency (IF) signal is first mixed with the local generated carrier and then correlated with the local pseudorandom noise (PRN) code. The correlation results are used in a phase discriminator to obtain the phase error that contains noise. Subsequently, the LF filters noisy phase error and generates a NCO control signal to synchronize the local carrier with the received signals. Relative dynamic and signal $C / N_{0}$ estimations are used to calculate the optimal loop gain in order to minimize the dominating tracking error.

The 1st- and 2nd-order loop filter and inertial element are all discredited to their digital forms by bilinear transformation, which are derived as follows:

$$
\begin{aligned}
& F_{1}(z)=\left.F_{1}(s)\right|_{s=\left(2 / T_{\mathrm{coh}}\right)\left(1-z^{-1} / 1+z^{-1}\right)}=\frac{b_{0}+b_{1} z^{-1}}{1-z^{-1}}, \\
& F_{2}(z)=\left.F_{2}(s)\right|_{s=\left(2 / T_{\mathrm{coh}}\right)\left(1-z^{-1} / 1+z^{-1}\right)}=\left(\frac{c_{0}+c_{1} z^{-1}+c_{2} z^{-2}}{1-2 z^{-1}+z^{-2}}\right), \\
& I(z)=\left.I(s)\right|_{s=\left(2 / T_{\mathrm{coh}}\right)\left(1-z^{-1} / 1+z^{-1}\right)}=\frac{T_{\mathrm{coh}}+T_{\mathrm{coh}} z^{-1}}{2 T+T_{\mathrm{coh}}+\left(T_{\mathrm{coh}}-2 T\right) z^{-1}},
\end{aligned}
$$

where

$$
\begin{aligned}
& b_{0}=\frac{1}{K}\left(2 \xi \omega_{n 1}+\frac{1}{2} \omega_{n 1}^{2} T_{\mathrm{coh}}\right), \\
& b_{1}=\frac{1}{K}\left(-2 \xi \omega_{n 1}+\frac{1}{2} \omega_{n 1}^{2} T_{\mathrm{coh}}\right), \\
& c_{0}=b \omega_{n 2}+\frac{1}{2} a \omega_{n 2}^{2} T_{\mathrm{coh}}+\frac{1}{4} T_{\mathrm{coh}}^{2} \omega_{n 2}^{3}, \\
& c_{1}=-2 b \omega_{n 2}+\frac{1}{2} T_{\mathrm{coh}}^{2} \omega_{n 2}^{3}, \\
& c_{2}=b \omega_{n 2}-\frac{1}{2} a \omega_{n 2}^{2} T_{\mathrm{coh}}+\frac{1}{4} T_{\mathrm{coh}}^{2} \omega_{n 2}^{3},
\end{aligned}
$$




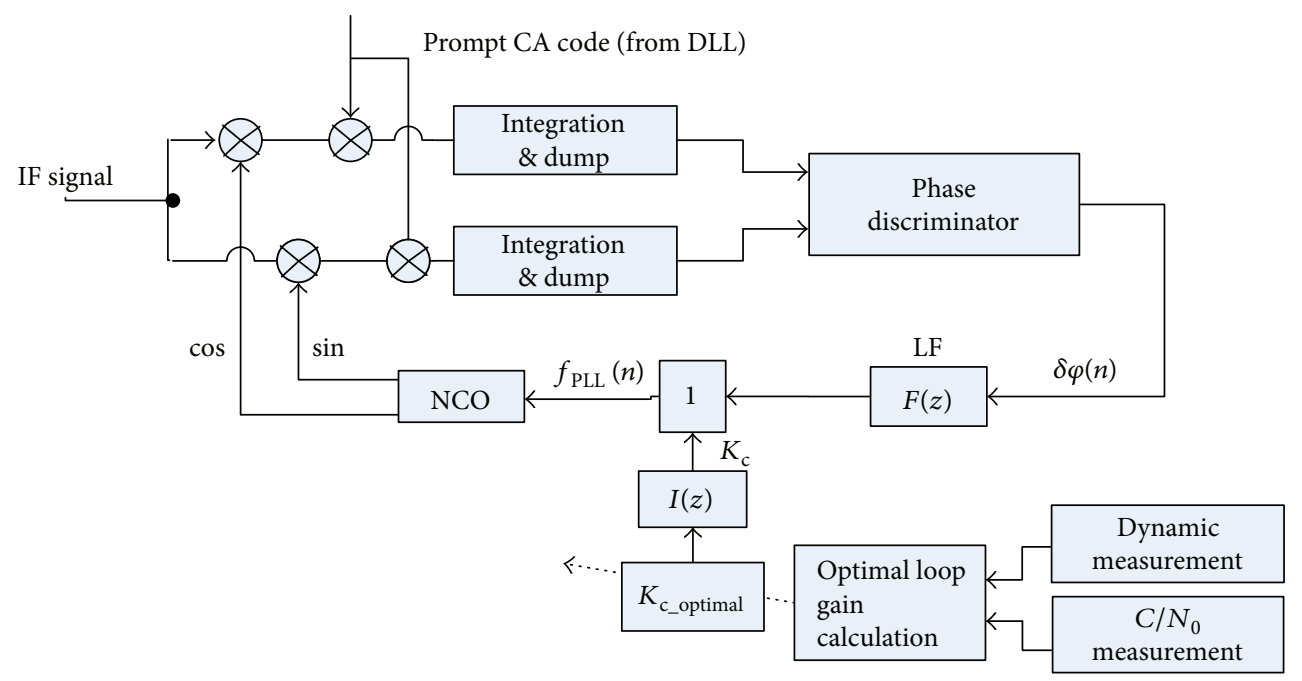

FIGURE 5: Digital implement of AGCF-PLL.

$T_{\text {coh }}$ is the coherent integration time in the unit of seconds. Arc tangent function was chosen as the phase discriminator in this paper.

\section{Time Domain and Frequency Domain Analyses of AGCF-PLL}

In this section, AGCF-PLL's behavior is analyzed in the time domain and frequency domain using the models derived in Section 2. The relationship between the AGCF-PLL's behavior and $K_{c}$ will be discussed in detail without considering the inertial element. The reason has been explained in Section 2. An averaging method of estimating relative acceleration and jerk will be derived based on time domain analysis. In the following discussion, the initial values of loop coefficients of the 2nd- and 3rd-order AGCF-PLL are chosen to be their common values as follows:

$$
\begin{aligned}
B_{n 1} & =20 \mathrm{~Hz}, \\
\xi & =0.707, \\
\omega_{n 1} & =\frac{8 \xi B_{n 1}}{4 \xi^{2}+1}=37.7142 \mathrm{~Hz}, \\
B_{n 2} & =18 \mathrm{~Hz}, \\
a & =1.1, \\
b & =2.4, \\
\omega_{n 2} & =\frac{4(a b-1)}{a b^{2}+a^{2}-b} B_{n 2}=22.9460 \mathrm{~Hz} .
\end{aligned}
$$

These loop coefficients can be adjusted by changing $K_{c}$, as discussed in Section 2.

3.1. Time Domain Analysis. The primary function of PLL is to make the local replicated carrier phase track the received one [16]. Therefore, the PLL could be regarded as a control system, or more specifically, a servo control system. Furthermore, the PLL system is a nonlinear feedback control system

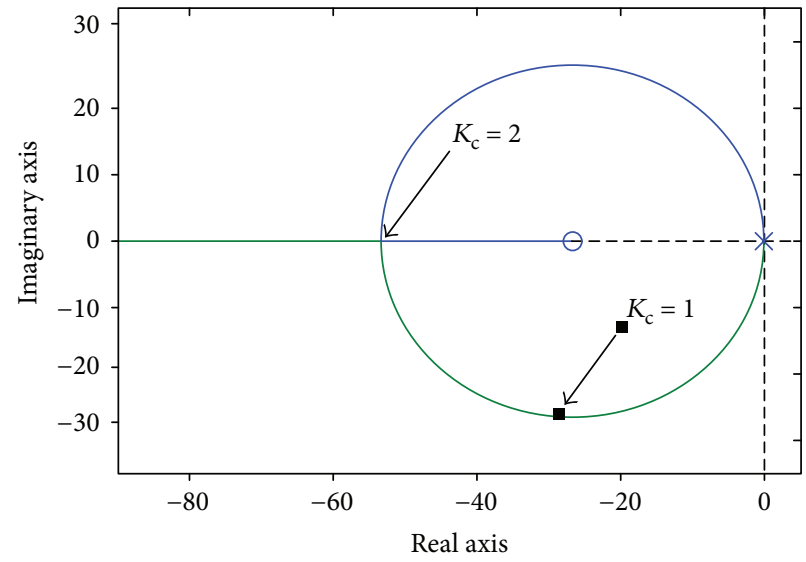

FIGURE 6: Root locus of the 2nd-order AGCF-PLL.

in the classical control theory point of views, where the loop filter is the controller and NCO is the plant. Its nonlinearity results from the nonlinear phase discriminator. Fortunately, the PLL's behavior in a locking state can be predicted by its linearized model, as derived in Section 2. Therefore, some classical analysis methods of the control system can be employed to analyze the AGCF-PLL. According to (8) and (10), the characteristic equation of the second- and thirdorder AGCF-PLL can be found as

$$
\begin{array}{r}
s^{2}+2 \xi \omega_{n 1} K_{c} s+\omega_{n 1}^{2} K_{c}=0 \\
s^{3}+b \omega_{n 2} K_{c} s^{2}+a \omega_{n 2}^{2} K_{c} s+\omega_{n 2}{ }^{3} K_{c}=0 .
\end{array}
$$

Figures 6 and 7 show the root locus of the 2nd- and 3rd-order AGCF-PLL for different $K_{c}$. It can be seen that with an increase in $K_{c}$, the closed-loop poles move towards minus infinity or open-loop zero points from open-loop poles. With the help of the classical control theory, it can be concluded that the response of the AGCF-PLL would be fast with less sinusoidal oscillation and less overshoot with $K_{\mathrm{c}}$ increment. It is worth noting that the 2nd-order AGCF-PLL is unconditionally stable while the 3rd-order 


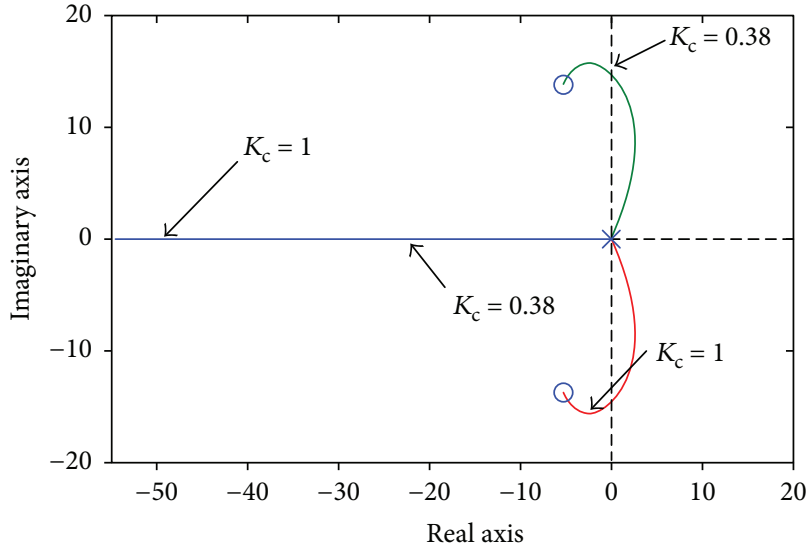

Figure 7: Root locus of the 3rd-order AGCF-PLL.

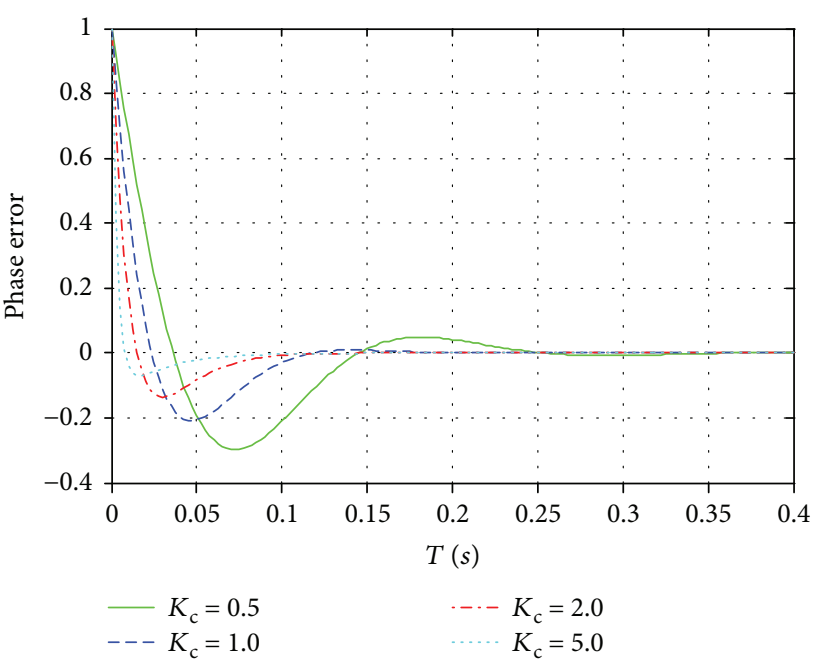

Figure 8: Step response of the 2nd-order AGCF-PLL.

AGCF-PLL is not. The stable condition of the third-order AGCF-PLL is

$$
K_{\mathrm{c}}>0.38
$$

Figures 8 and 9 show the step response of the AGCF-PLL for different values of the loop gain. The results of step response simulation are consistent with those of the previous analysis and more visualized.

The PLL tracking error caused by relative dynamics could be written as [14]

$$
\theta_{e}=\frac{1}{\lambda \omega_{n}^{N}} \frac{d^{N} R}{d t^{N}}
$$

where $N$ is the order of PLL, $\theta_{e}$ is the tracking error in the unit of meters, and $\lambda$ is the carrier wavelength in the unit of meters. According to (13), (15), and (24), increasing $K_{c}$ can decrease dynamic stress error. In conclusion, large $K_{\mathrm{c}}$ brings about high convergence speed and less dynamic stress error. Thus, large $K_{c}$ is expected in the time domain without regard to the noise in the input carrier.

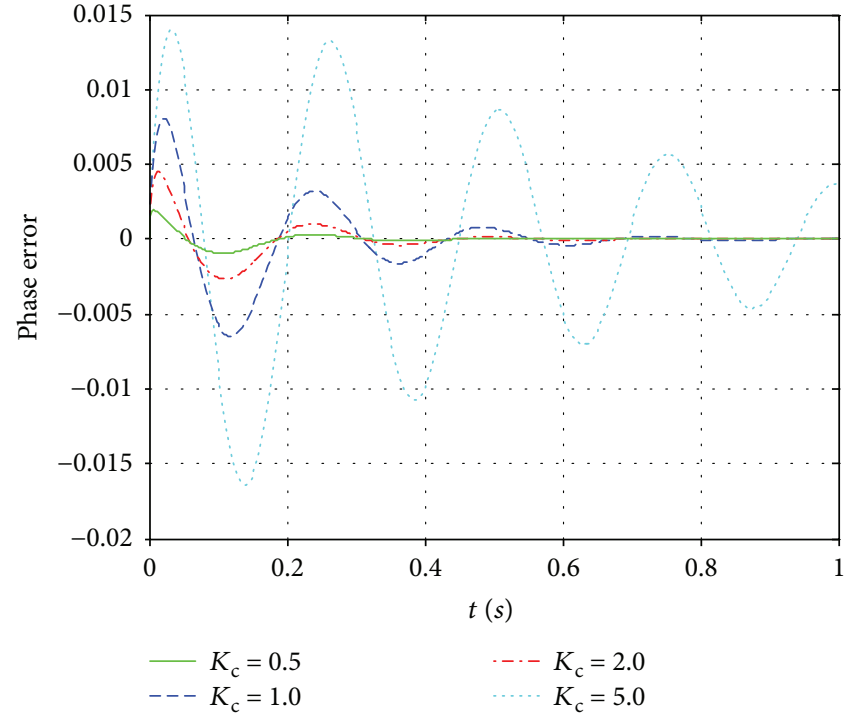

FIgUre 9: Step response of the 3rd-order AGCF-PLL.

3.2. Frequency Domain Analysis. The PLL tracks the noisy received carrier phase so that the local replicated carrier contains noise unavoidably. Therefore, the secondary function of PLL is to filter the noise in the received carrier phase in order to obtain an accurate carrier replica. As one of the important parameters of the PLL, noise bandwidth controls the amount of noise allowed in the PLL. This parameter is defined as follows [15]:

$$
B_{n}=\frac{1}{2 \pi} \int_{0}^{\infty}|H(j \omega)| d f .
$$

The relationship between the noise bandwidths and loop coefficients for the 2nd- and 3rd-order PLL can be expressed as follows:

$$
\begin{aligned}
& B_{n 1}=\frac{\omega_{n 1}}{2}\left(\xi+\frac{1}{4 \xi}\right), \\
& B_{n 2}=\frac{a b^{2}+a^{2}-b}{4(a b-1)} \omega_{n 2},
\end{aligned}
$$

where $B_{n 1}$ and $B_{n 2}$ are the loop noise bandwidths of 2nd- and 3rd-order PLL, respectively.

The relationship between the noise bandwidth and $K_{c}$ of the 2nd-order AFCF-PLL can be derived by inserting (12) and (13) into (26). Similarly, this relationship for the 3rd-order AGCF-PLL can be derived by inserting (15), (16), and (17) into (27). Finally, those two relationships are expressed as follows:

$B_{n 1}^{\prime}=\frac{\xi \omega_{n 1}}{2} \cdot K_{\mathrm{c}}+\frac{\omega_{n 1}}{8 \xi}=13.3319 K_{\mathrm{c}}+6.6680$,

$B_{n 2}^{\prime}=\frac{K_{c}\left(K_{c} a b^{2}+a^{2}-b\right) \omega_{n 2}}{4\left(K_{c} a b-1\right)}=\frac{145.3858 K_{c}^{2}-1.19}{10.56 K_{c}-4}$. 


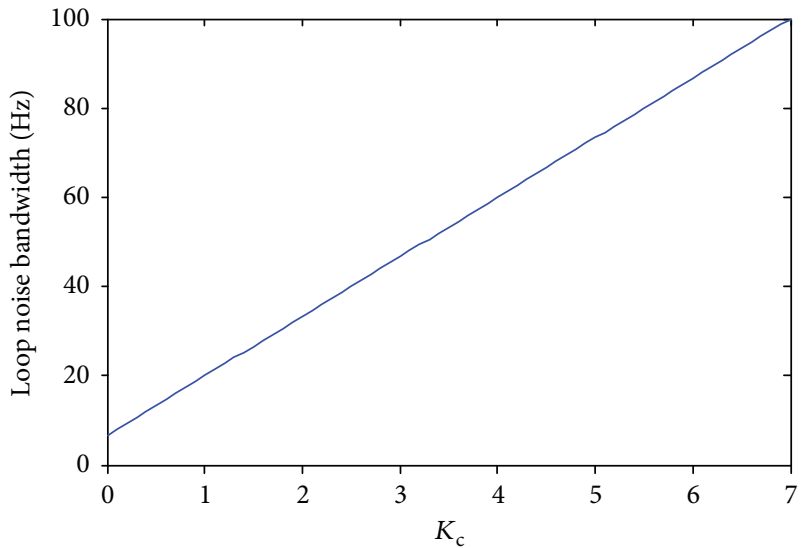

FIGURE 10: The relation between the loop noise bandwidth and loop gain for the 2nd-order AGCF-PLL.

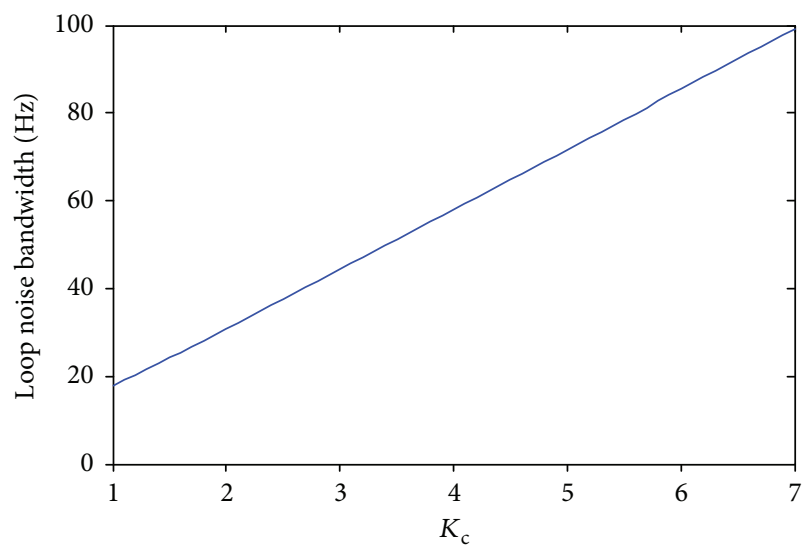

Figure 11: The relation between the loop noise bandwidth and loop gain for the 3rd-order AGCF-PLL.

Here, $B_{n 1}^{\prime}$ and $B_{n 2}^{\prime}$ are the loop noise bandwidths of the 2nd- and 3rd-order AGCF-PLL, respectively.

Figures 10 and 11 show the relationship between the loop noise bandwidth and $K_{\mathrm{c}}$ for the AGCF-PLL in graphic way. It can be concluded that the loop noise bandwidth of the 2nd order AGCF-PLL is proportional to the $K_{c}$ while the loop noise bandwidth of the 3rd order AGCF-PLL is positively correlated with the $K_{\mathrm{c}}$.

3.3. The Estimation of Relative Dynamic and $C / N_{0}$. As the first step to calculate optimal $K_{c}$, relative acceleration and jerk estimations play important roles in AGCF-PLL. Actually, (24) provides us a method to calculate relative acceleration or jerk according to phase error. As long as the AGCFPLL is in a locking state, the relative acceleration can be obtained from the 2nd-order AGCF-PLL and the relative jerk can be obtained from the 3rd-order AGCF-PLL. This relation is shown as follows:

$$
\begin{aligned}
\operatorname{Acceleration}(n) & =\theta_{e 1}(n) \lambda \omega_{n 1}^{\prime}, \\
\operatorname{Jerk}(n) & =\theta_{e 2}(n) \lambda \omega_{n 2}^{\prime},
\end{aligned}
$$

where $\theta_{e 1}(n)$ is the $n$th phase error value estimated by the 2nd-order AGCF-PLL and $\theta_{e 2}(n)$ is the $n$th phase error value estimated by the 3rd-order AGCF-PLL. However, the estimated phase error contains much noise, which results in noisy acceleration and jerk estimations. Therefore, a simple averaging operation is employed to filter the noise. Thus, (29) can be rewritten as follows:

$$
\begin{aligned}
\text { Acceleration }(k) & =\lambda \omega_{n 1}^{\prime} \frac{1}{M} \sum_{n=k M+1}^{k M+M+1} \theta_{e 1}(n), \\
\operatorname{Jerk}(k) & =\lambda \omega_{n 2}^{\prime} \frac{1}{N} \sum_{n=k M+1}^{k M+M+1} \theta_{e 2}(n),
\end{aligned}
$$

where $M$ is the number of phase error values from a phase discriminator. $M$ should not be too small considering the noise influence. In this paper, $N$ is set as 500 .

$\mathrm{C} / \mathrm{N}_{0}$ estimations are another coefficient that the AGCFPLL algorithms need to calculate the optimal $K_{\mathrm{c}}$. The correlator comparison method is used to measure $C / N_{0}$ in this paper [18]. The measurement, $Z$, is first formed as follows:

$$
Z=\frac{\sum_{i=1}^{L} I_{P}^{2}(i)+Q_{P}^{2}(i)}{2 \sum_{i=1}^{M} I_{N}^{2}(i)}
$$

where $I_{P}$ and $Q_{P}$ are the accumulated outputs of the prompt correlator, $I_{N}$ is the unsynchronized accumulated correlator output, and $L$ denotes the number of iterations averaged over to smooth noise. Then, $C / N_{0}$ estimations could be calculated employing the following equation:

$$
\begin{aligned}
\frac{c}{n_{0}} & =\frac{1}{T_{\operatorname{coh}}}\left(\frac{L}{L+2} Z-1\right), \\
\frac{C}{N_{0}} & =10 \cdot \lg \frac{c}{n_{0}},
\end{aligned}
$$

where $C / N_{0}$ is in the unit of $\mathrm{dB}-\mathrm{Hz}$ while $c / n_{0}$ is in the unit of $\mathrm{Hz}$.

\section{Optimized Loop Gain of AGCF-PLL}

In this section, the AGCF-PLL measurement error will be derived and discussed in different conditions first. Then, the optimal $K_{\mathrm{c}}$ is calculated according to the estimated $C / N_{0}$ and relative dynamic to obtain the minimum tracking error. Finally, the loop gain control method will be proposed.

4.1. Error Characteristic of AGCF-PLL. The dominant sources of the phase error in the PLL are phase jitter and dynamic stress error. The phase jitter contains thermal noise and oscillator noise (i.e., vibration-induced oscillator jitter and Allan variance-induced oscillator jitter) [15]. The 1- $\sigma$ tracking error of the PLL is given by 


$$
\begin{aligned}
\delta_{\mathrm{PLL}}\left(B_{n}, \frac{d^{N} R}{d t^{N}}, \frac{c}{n_{0}}\right)= & \delta_{t \mathrm{PLL}}+\frac{\theta_{e}}{3} \\
= & \frac{180}{\pi} \sqrt{\frac{B_{n}}{c / n_{0}}\left(1+\frac{1}{2 T_{\mathrm{coh}} \cdot c / n_{0}}\right)} \\
& +\frac{1}{3} \frac{1}{\left(\omega_{n}\right)^{2}} \frac{d^{N} R}{d t^{N}} \frac{360}{\lambda}
\end{aligned}
$$

where $R$ is the line-of-sight (LOS) distance between the satellite and receiver in the unit of meters. $T_{\text {coh }}$ is chosen to be 1 millisecond in this paper. Vibration-induced oscillator jitter (approximately $0.5^{\circ}$ ) and Allen variance-induced oscillator jitter (approximately $2^{\circ}$ ) are also considered but not expressed in the equation for the sake of simplicity.

The 1- $\sigma$ tracking error of the 2nd-order AGCF-PLL could be derived by inserting (12) and (13) into (33):

$$
\begin{aligned}
\delta_{\mathrm{PLL1}}\left(K_{\mathrm{c}}, \frac{d^{2} R}{d t^{2}}, \frac{c}{n_{0}}\right) \\
=\delta_{t \mathrm{PLL}}+\frac{\theta_{e}}{3} \\
=\frac{180}{\pi} \sqrt{\frac{\xi \omega_{n 1} / 2 \cdot K_{\mathrm{c}}+\left(\omega_{n 1} / 8 \xi\right)}{c / n_{0}}\left(1+\frac{1}{2 T_{\mathrm{coh}} \cdot c / n_{0}}\right)} \\
+\frac{1}{3} \frac{1}{\left(\sqrt{K_{\mathrm{c}}} \omega_{n 1}\right)^{2}} \frac{d^{2} R}{d t^{2}} \frac{360}{\lambda} .
\end{aligned}
$$

Similarly, the 1- $\sigma$ tracking error of the 3rd-order AGCF-PLL could be derived by inserting (15), (16), and (17) into (33):

$$
\begin{aligned}
\delta_{\mathrm{PLL}}\left(K_{\mathrm{c}}, \frac{d^{3} R}{d t^{3}}, \frac{c}{n_{0}}\right) \\
=\delta_{t \mathrm{PLL}}+\frac{\theta_{e}}{3} \\
=\frac{180}{\pi} \sqrt{\frac{K_{\mathrm{c}}\left(K_{\mathrm{c}} a b^{2}+a^{2}-b\right) \omega_{n 2} / 4\left(K_{\mathrm{c}} a b-1\right)}{c / n_{0}}\left(1+\frac{1}{2 T_{\mathrm{coh}} \cdot c / n_{0}}\right)} \\
+\frac{1}{3} \frac{1}{\left(\sqrt[3]{K_{\mathrm{c}}} \omega_{n 2}\right)^{2}} \frac{d^{3} R}{d t^{3}} \frac{360}{\lambda} .
\end{aligned}
$$

The error characteristic with an acceleration of $4 g$ and different $C / N_{0}$ is shown in Figure 12, where $g$ is the acceleration of the gravity. It can be observed that the 1- $\sigma$ tracking error decreases to minimum, then increases to positive infinity with the increase in $K_{c}$. So there exists minimum, maximum, and optimum $K_{\mathrm{c}}$. The maximal and minimal values were located at the crossing point of the error characteristic curve and tracking threshold curve $\left(15^{\circ}\right)$. The optimum $K_{\mathrm{c}}$ is located on the point that has the minimal phase error. It could also be observed that the high $K_{\mathrm{c}}$ would extend the range of $C / N_{0}$. The error characteristic of the 3rd-order

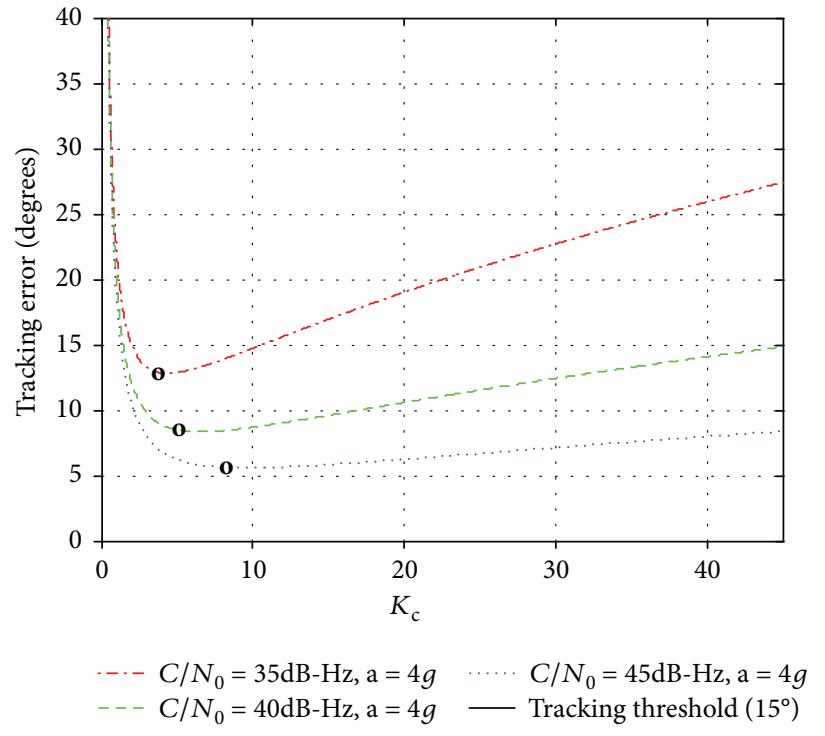

FIgURe 12: Error characteristic of the 2nd-order AGCF-PLL.

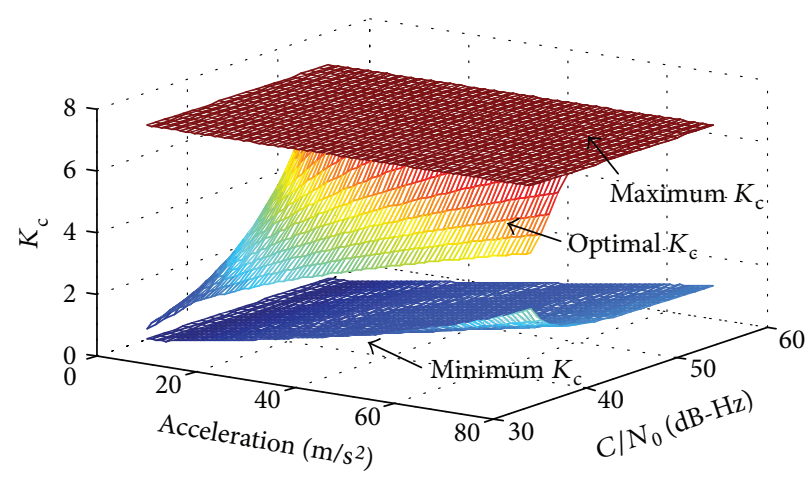

FIgURE 13: The maximum, minimum, and optimal $K_{\mathrm{c}}$ for the 2 ndorder AGCF-PLL.

AGCF-PLL is similar to that of the 2nd-order AGCF-PLL and sensitive to relative jerk, which is not shown here.

4.2. Optimized Loop Gain of AGCF-PLL. According to the analysis above, the optimal value of $K_{c}$ could be calculated by (36) while the maximum and minimum values of $K_{c}$ could be calculated by (37). Because high $B_{n} T_{\text {coh }}$ would degrade the loop performance when the transformation (bilinear, boxcar transform) method is used to implement the loop filter $[19,20]$, another constraint $\left(B_{n} T_{\text {coh }}<0.1\right)$ is added.

$$
\begin{aligned}
\frac{\partial \delta_{\mathrm{PLL}}}{\partial K_{\mathrm{c}}} & =0, \\
\delta_{\mathrm{PLL}} & =15^{\circ}, \\
B_{n}^{\prime} T_{\mathrm{coh}} & <0.1 .
\end{aligned}
$$

Figures 13 and 14 show the maximum, minimum, and optimal $K_{\mathrm{c}}$ of the 2nd- and 3rd-order AGCF-PLL in different conditions. Figures 15 and 16 show their minimal tracking errors in different conditions when optimal $K_{\mathrm{c}}$ is employed. 


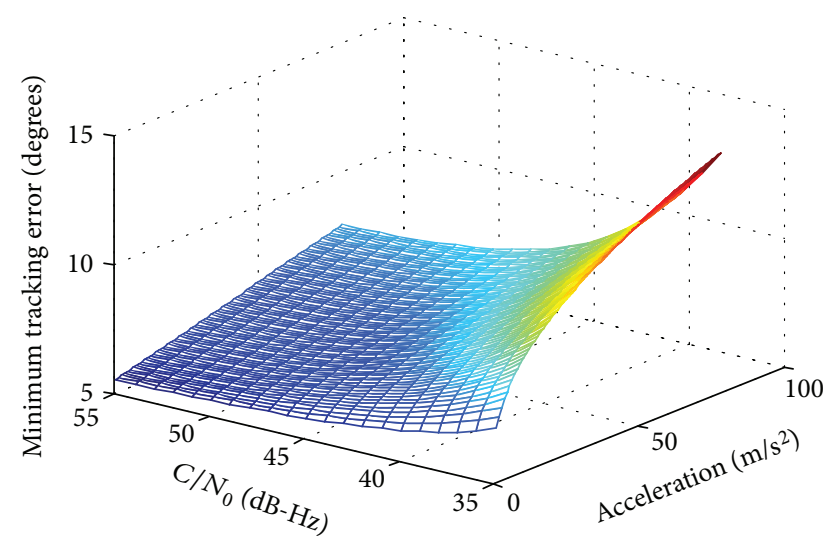

FIGURE 14: The maximum, minimum, and optimal loop gain for the 3rd-order AGCF-PLL in different conditions.

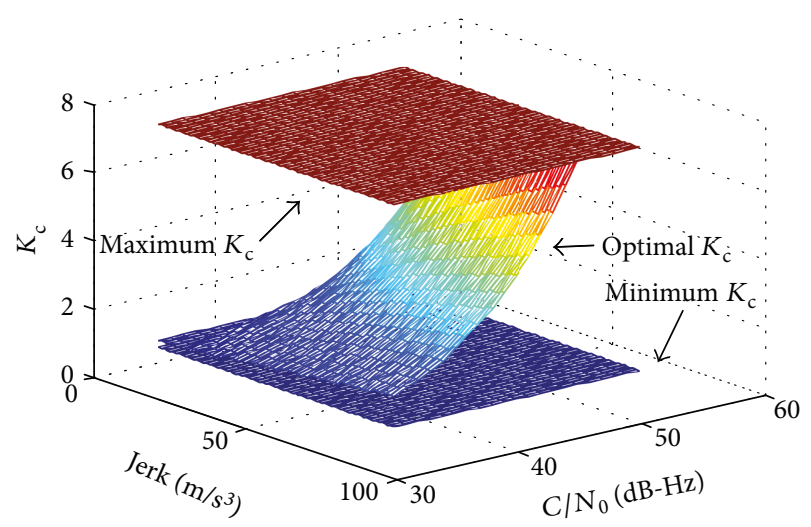

FIgURE 15: The minimum tracking error using optimal $K_{\mathrm{c}}$ for the2nd order AGCF-PLL.

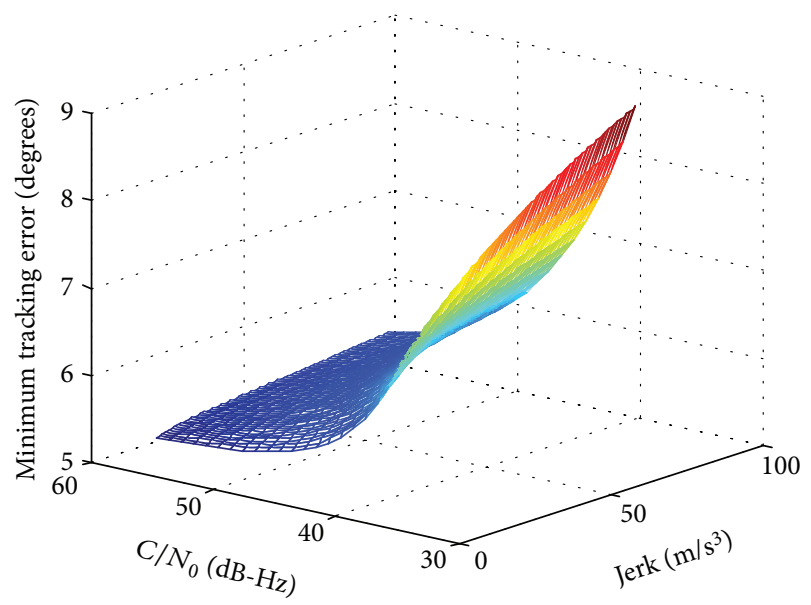

Figure 16: The minimum tracking error using the optimal loop gain for the 3rd-order AGCF-PLL.

4.3. Adaptive Loop Gain Control Strategy. Based on the above analysis, the maximal $K_{c}$ could be used initially to make the tracking loop converge from the pull-in state to the locking state. The reason has been discussed in Section 3. After the loop goes into the locking state, $K_{\mathrm{c}}$ can be adjusted adaptively
TABLE 1: The lookup table for the second-order AGCF-PLL $\left(\mathrm{C} / \mathrm{N}_{0}=43 \mathrm{~dB}-\mathrm{Hz}\right)$.

\begin{tabular}{lccc}
\hline Acceleration & Minimum $K_{c}$ & $\begin{array}{c}\text { The loop gain } \\
\text { Optimal } K_{c}\end{array}$ & Maximum $K_{c}$ \\
\hline$g$ & 0.2 & 3.5 & 7 \\
$2 g$ & 0.5 & 5 & 7 \\
$3 g$ & 0.7 & 6.2 & 7 \\
$4 g$ & 0.9 & 7 & 7 \\
$5 g$ & 1.2 & 7 & 7 \\
$6 g$ & 1.4 & 7 & 7 \\
$7 g$ & 1.6 & 7 & 7 \\
$8 g$ & 1.9 & 7 & 7 \\
\hline
\end{tabular}

TABle 2: The lookup table for the third-order AGCF-PLL $\left(\mathrm{C} / \mathrm{N}_{0}=45 \mathrm{~dB}-\mathrm{Hz}\right)$.

\begin{tabular}{lccc}
\hline Jerk & Minimum $K_{\mathrm{c}}$ & $\begin{array}{c}\text { The loop gain } \\
\text { Optimal } K_{\mathrm{c}}\end{array}$ & Maximum $K_{\mathrm{c}}$ \\
\hline $\mathrm{g} / \mathrm{s}$ & 0.5 & 1.5 & 7 \\
$2 \mathrm{~g} / \mathrm{s}$ & 0.5 & 2 & 7 \\
$3 \mathrm{~g} / \mathrm{s}$ & 0.5 & 2.5 & 7 \\
$4 \mathrm{~g} / \mathrm{s}$ & 0.5 & 2.9 & 7 \\
$5 \mathrm{~g} / \mathrm{s}$ & 0.5 & 3.2 & 7 \\
$6 \mathrm{~g} / \mathrm{s}$ & 0.5 & 3.5 & 7 \\
$7 \mathrm{~g} / \mathrm{s}$ & 0.5 & 3.9 & 7 \\
$8 \mathrm{~g} / \mathrm{s}$ & 0.5 & 4.1 & 7 \\
\hline
\end{tabular}

according to the $C / N_{0}$ and relative dynamic estimations. Considering the complexity to solve (36) and (37) that have a radical sign, a lookup table is used to store the relationship among $C / N_{0}$ estimations, relative dynamic estimations, and optimal $K_{\mathrm{c}}$ in a discrete form. The $C / N_{0}$ and relative dynamic estimations discussed in Section 3 contain accumulation that could be considered to be the first low-pass filtering. The estimated $C / N_{0}$ and relative dynamic will be the input of the lookup table. The two-dimensional linear interpolation method is employed to calculate the optimal $K_{c}$ subsequently. However, the estimated $C / N_{0}$ and relative dynamic still contain noise. Thus, the optimal $K_{c}$ is noisy too. The inertial element introduced here would further filter the noise in optimal $K_{c}$. Therefore, the adjustment frequency decreases. A part of the lookup table with specific $C / N_{0}$ and dynamic for AGCF-PLL is shown in Tables 1 and 2.

\section{Test, Results, and Analysis}

The algorithms discussed in this paper were implemented in the software GPS receiver and tested using simulated GPS signals from a navigation satellite signal simulator. Because the 2nd-order AGCF-PLL is sensitive to relative acceleration while the 3rd-order AGCF-PLL is sensitive to relative jerk, two scenarios were designed to demonstrate the tracking performance under high-dynamic 


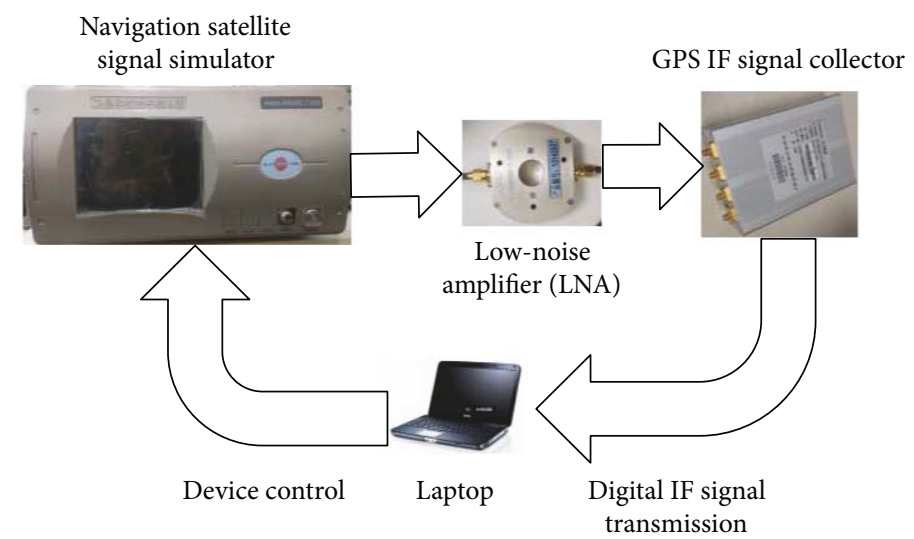

FIgURE 17: Experimental setup.
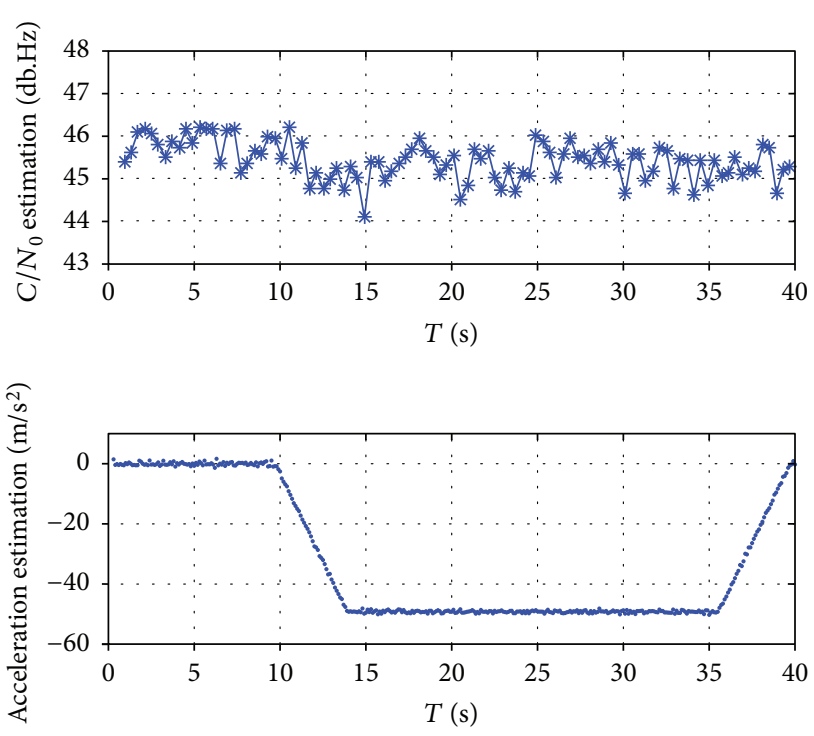

FIgURE 18: $C / N_{0}$ and acceleration estimations from the 2nd-order AGCF-PLL.

application-high-acceleration scenario and high-jerk scenario. The AGCF-PLL is compared to their constant loop gain versions (conventional PLL), FLL-assisted PLL, and FAB-LL, to show the improvements.

5.1. Experimental Setup. In Figure 17, the navigation satellite signal simulator (HWA-RNSS-7300 manufactured by HWA Create Corporation Ltd.) controlled by a laptop can provide L1 C/A code signals under various scenarios. The complicated straight motion scenario is employed to generate high-dynamic motion. The power of the carrier signal can be adjusted by the laptop control signal, which can be used to change the $C / N_{0}$ of $\mathrm{C} / \mathrm{A}$ code signals. A low-noise amplifier (LNA; HLLNA012-1-02B) is used to play the same role as the LNA in a real GPS antenna. Connected with LNA is the GPS IF signal collector (NewStar210M made by OlinkStar Corporation) which can transfer digital IF signals to the laptop. At last, the postprocessing including acquisition and tracking for the IF signal is done by software.
5.2. Metrics. The metrics used to assess the algorithm performance is the cosine value of the phase difference $\left(\phi_{e}\right)$, which is given by

$$
\cos \phi_{e}=\frac{\left|I_{p}(n)\right|}{\sqrt{I_{p}(n)^{2}+Q_{p}(n)^{2}}} .
$$

When PLL is locked, the phase error should keep close to 0 and therefore $\cos \phi_{e}$ should be approximately equal to one. Moreover, $\cos \phi_{e}$ deviating from one indicates that PLL performance degrades, while the irregular alterations of $\cos \phi_{e}$ mean that the loop is losing lock. It is safe to assume that if the value of the lock indicator is greater than 0.8 , the PLL is locked [4]. Although this value is corrupted with noise, it is this value that indicated that the loop locking status and its mean value can be used to assessing carrier tracking performance of the loop.

5.3. Test Results and Analysis for the 2nd-Order AGCF-PLL. The high-acceleration scenario (test scenario A) is simulated and used to test the 2nd-order AGCF-PLL algorithms, and its performance is compared with the traditional 2nd-order ordinary PLL, 1st-order FLL-assisted 2nd-order PLL, and FAB-LL. At the beginning, the vehicle stays for 10 seconds and then a high-acceleration motion is simulated. The bandwidth of the ordinary 2nd-order PLL is set to $20 \mathrm{~Hz}$. The bandwidth of FLL and PLL for FLL-assisted PLL is set to $5 \mathrm{~Hz}$ and $20 \mathrm{~Hz}$, respectively. The test GPS signal came from satellite 21.

In order to adjust the $K_{c}$, the $C / N_{0}$ and relative acceleration are estimated firstly, as shown in Figure 18. Compared with estimations given by [4], the averaging method makes the estimated acceleration less noisy, which will decrease the regulating frequency of $K_{\mathrm{c}}$. According to the estimated signal conditions, the optimal $K_{c}$ can be found through the lookup table and two-dimensional interpolation, as discussed in Section 4. Figure 19 shows the optimal and actual $K_{c}$. We can find that the actual $K_{\mathrm{c}}$ would track the optimal $K_{\mathrm{c}}$ in a slow and smooth way, avoiding PLL losing lock which is caused by sharp $K_{\mathrm{c}}$ adjustment. 


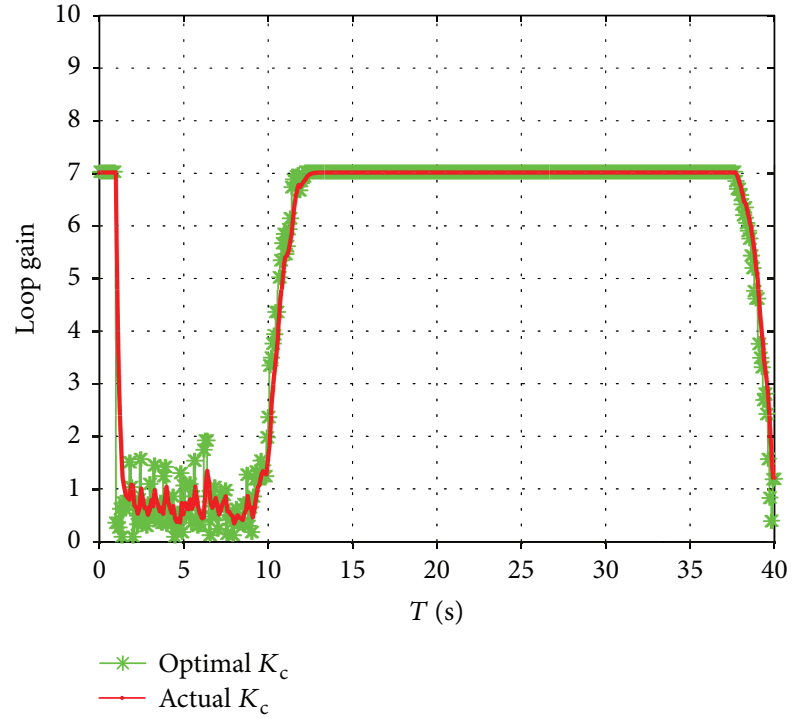

FIGURE 19: The optimal and actual loop gain of the 2nd-order AGCF-PLL.

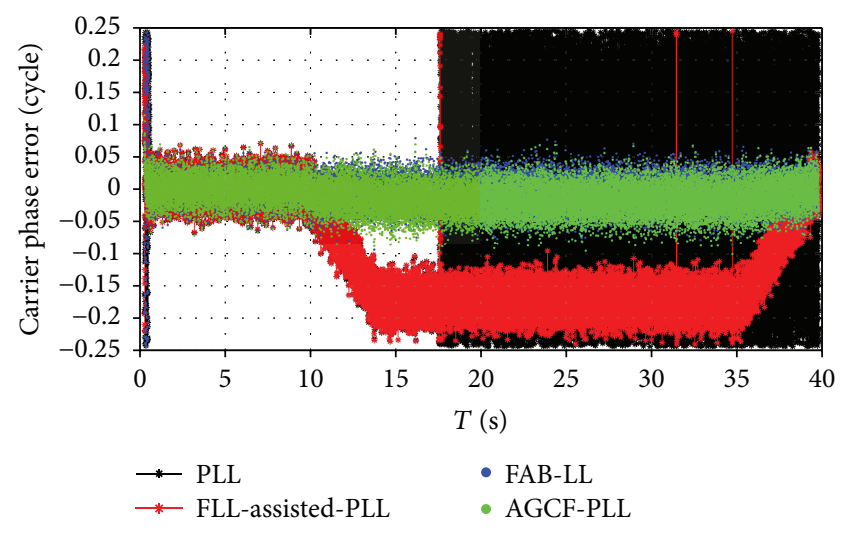

FIgURE 20: The arc tangent discriminator outputs of the four methods in test scenario A.

Figure 20 shows the arc tangent discriminator outputs of the four kinds of PLL. The arc tangent discriminator outputs represent the carrier phase tracking error. It can be found that the ordinary 2nd-order PLL cannot bear the highacceleration motion and lost lock for the carrier phase eventually. The 1st-order FLL-assisted 2nd-order PLL is able to track the carrier phase dynamic. However, there is a steadystate error. The carrier phase tracking error is very close to the effective working range $(-0.25$ cycle, 0.25 cycle) of the arc tangent discriminator. Thus, this PLL is easy to lose lock, especially when relative acceleration or noise increases. As for the FAB-LL and AGCF-PLL, they can keep the carrier phase lock in the high-acceleration scenario with less carrier phase tracking error. The AGCF-PLL has comparable performance with FAB-LL according to this figure. In order to quantitatively evaluate their performance, further evaluation is done and shown as follows.

Figure 21 shows the PLL lock status of the four kinds of PLL in test scenario A, while Table 3 presents the mean value

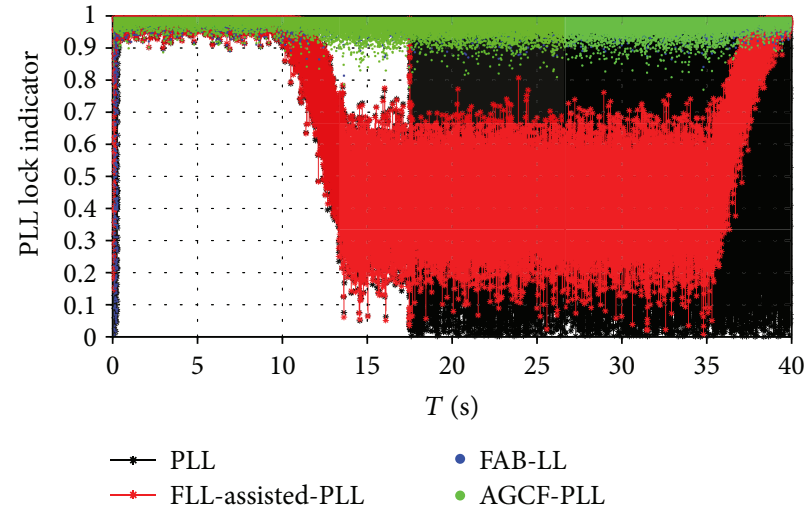

FIgURE 21: PLL lock indicator of the four methods in test scenario A.

TABLE 3: Mean value of the PLL lock indicator of the four methods in test scenario $\mathrm{A}$.

\begin{tabular}{lc}
\hline Method & Mean value of the PLL lock indicator \\
\hline PLL & - \\
FLL-assisted PLL & 0.6456 \\
FAB-LL & 0.9841 \\
AGCF-LL & 0.9883 \\
\hline
\end{tabular}

of the lock indicator. Because the ordinary PLL loses lock for the carrier phase in test scenario $\mathrm{A}$, the mean value of the lock indicator is invalid and not shown in this table. The mean value of the lock indicator of the AGCF-LL during highacceleration motion (10 40 s) is about 0.9883 , which is closer to 1 than that of the FAB-LL. This suggests that the 2ndorder AGCF-PLL outperforms the other kinds of 2nd-order PLL in high-acceleration scenarios.

5.4. Test Results and Analysis for the 3rd-Order AGCF-PLL. Similarly, a high-jerk scenario (test scenario B) is simulated and employed to test the performance of the ordinary 3rdorder PLL, 2nd-order FLL-assisted 3rd-order PLL, 3rdorder FAB-LL, and 3rd-order AGCF-PLL. The bandwidth of the ordinary 3rd-order PLL is set to $18 \mathrm{~Hz}$. The bandwidth of FLL and PLL for FLL-assisted PLL is set to $5 \mathrm{~Hz}$ and $18 \mathrm{~Hz}$, respectively. The test GPS signal came from satellite 31 .

Figure 22 shows the estimated $C / N_{0}$ and relative jerk of AGCF-PLL in test scenario $B$. These two data determine the optimal $K_{\mathcal{c}}$, which is able to minimize the carrier phase tracking error. The inertial element is employed to make the actual $K_{\mathrm{c}}$ track the optimal $K_{\mathrm{c}}$ in a smooth way, thus decreasing the effect of noisy $C / N_{0}$ estimation and relative jerk estimation. This relation is shown in Figure 23.

Figure 24 shows the arc tangent discriminator outputs of the four kinds of PLL in test scenario B. The four kinds of PLL are all able to track the carrier phase dynamic. However, the carrier phase tracking errors of the ordinary 3rd-order PLL and 2rd-order FLL-assisted 3rd-order PLL are bigger than those of AGCF-PLL and FAB-LL. The FAB-LL and AGCF-PLL can keep the carrier phase lock in the highjerk scenario with less carrier phase tracking error. The 

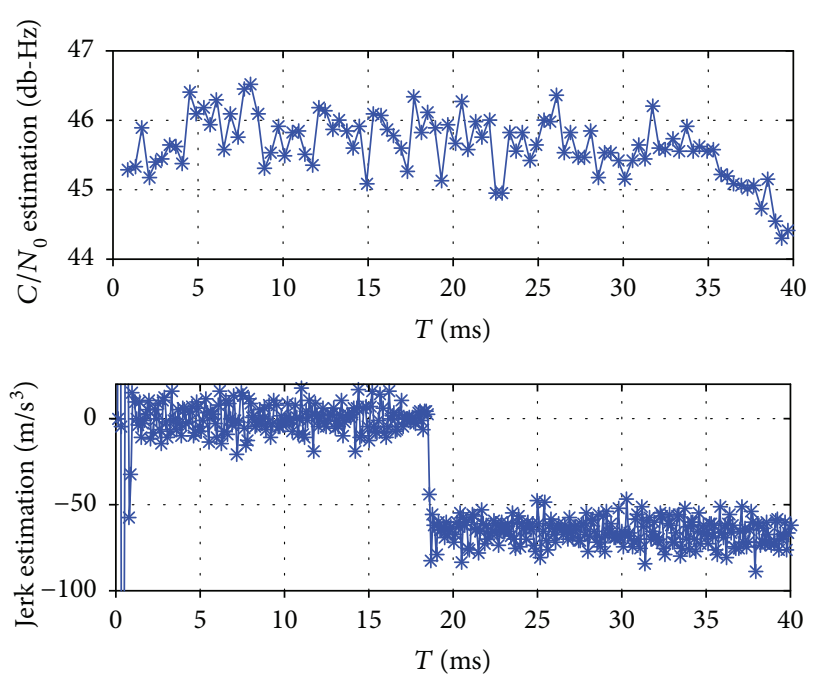

Figure 22: $C / N_{0}$ and acceleration estimations from the 3rd-order AGCF-PLL.

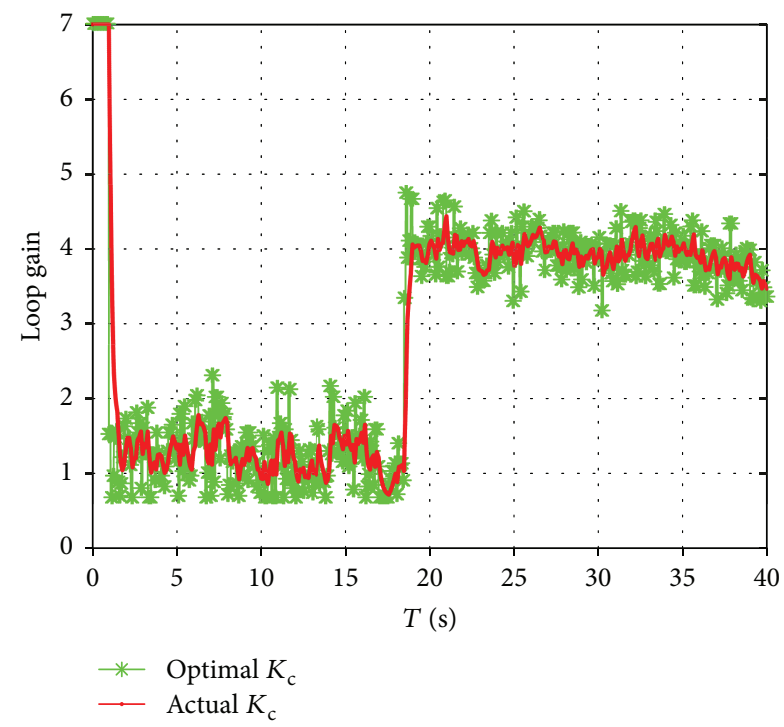

FIGURE 23: The optimal and actual loop gain of the 3rd-order AGCF-PLL.

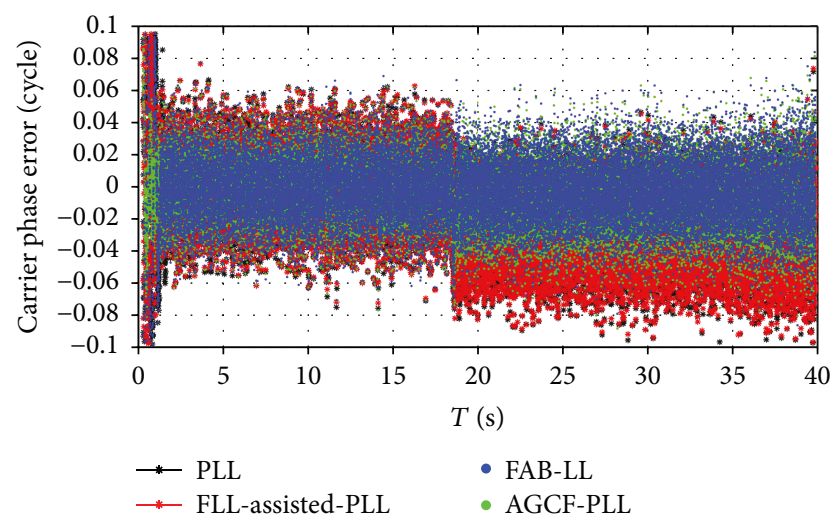

FIgURE 24: The arc tangent discriminator outputs of the four methods in test scenario B.

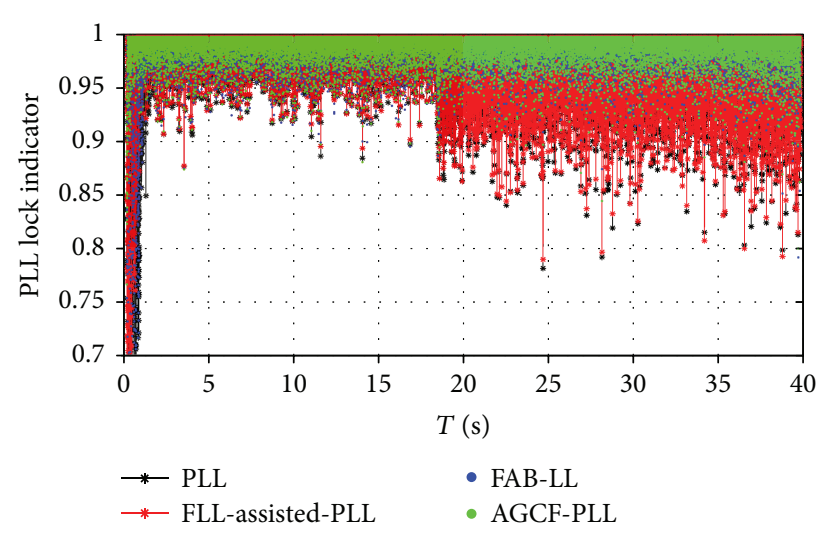

FIGURE 25: PLL lock indicator of the four methods in test scenario B.

TABLE 4: Mean value of the PLL lock indicator of the four methods in test scenario $\mathrm{B}$.

\begin{tabular}{lc}
\hline Method & Mean value of the PLL lock indicator \\
\hline PLL & 0.9784 \\
FLL-assisted PLL & 0.9813 \\
FAB-LL & 0.9868 \\
AGCF-LL & 0.9916 \\
\hline
\end{tabular}

AGCF-PLL has comparable performance with FAB-LL according to this figure. In order to quantitatively evaluate their performance, we did further evaluation and the results are shown as the following parts.

Figure 25 shows the PLL lock status of the four kinds of PLL in test scenario B, while Table 4 presents the mean value of the lock indicator. The mean value of the lock indicator of 3rd-order AGCF-PLL during high-jerk motion (18 40 s) is about 0.9916 , which is closest to 1 , than that of other kinds of 3rd-order PLL. This suggests that 3rd-order AGCF-PLL has the least carrier phase tracking error in these four kinds of PLL.

\section{Conclusions}

This paper presents a novel adaptive loop gain control approach for second- and third-order PLL in GNSS receivers, and its performance was tested and verified compared with that of the traditional PLL. The new system model, time and frequency domain analyses, and error characteristic of the new PLL are derived and displayed in detail, which could be regarded as the first contribution of this paper. Although only GPS L1 C/A code signals are tested, it is easy to generalize this method to other GNSS signals. The loop gain can be adjusted to its optimal value according to $C / N_{0}$ and relative dynamic estimations to obtain the minimal tracking error. Compared with the conventional PLL, FLL-assisted PLL, FAB-LL, the proposed adaptive loop gain algorithms have a stronger tracking ability to deal with high-dynamic stress.

An inertial element is inserted in the new adaptive PLL in order to decrease the change rate of the loop gain, whose intense adjustment may cause a disturbance to PLL or results in PLL losing lock for the carrier phase. 
The third contribution of this paper is the lookup table which stores the relationships among the signal conditions and optimal loop gain. Using the method of the lookup table to calculate the loop gain decreases the computation cost depending on calculating equation with radicals so that a real-time AGCF-PLL can be realized.

The predetection integration time and loop order are another two coefficients of PLL that can be made adaptive. These two coefficients can also be adjusted according to signal condition to increase the tracking performance of PLL. But the predetection integration time adjustment and loop order adjustment are not discussed in this paper and would become the next research orientation.

\section{Conflicts of Interest}

The authors declare that they have no conflicts of interest.

\section{Acknowledgments}

This work is funded by the National Natural Science Foundation of China under Grant nos. 61633008, 61304234, 61773132, and 61374007.

\section{References}

[1] P. W. Ward, Performance Comparisons between FLL, PLL and a Novel FLL-Assisted-PLL Carrier Tracking Loop under RF Interference Conditions, Proceedings of the 11th International Technical Meeting of the Satellite Division of the Institute of Navigation (ION GPS 1998), Nashville, USA, 1998.

[2] S. Alban, D. M. Akos, S. M. Rock, and D. Gebre-Egziabher, Performance Analysis and Architectures for INS-Aided GPS Tracking Loops, Institute of Navigation-ITM (2003), San Diego, California, USA, 2003.

[3] P. Misra and P. Enge, Global Positioning System: Signals, Measurements and Performance, Ganga-Jamuna Press, Lincoln, MA, Second edition, 2011.

[4] P. Lian, Improving tracking performance of PLL in high dynamic applications, [Ph.D. thesis], Department of Geomatics Engineering University of Calgary, Calgary, Canada, 2004.

[5] P. Rinder, Design of a single frequency GPS software receiver, [Ph.D. thesis], Institute of Electronic Systems, Aalborg University, Aalborg, Denmark, 2004.

[6] F. Legrand, C. Macabiau, J. L. Issler, L. Lestarquit, and C. Mehlen, Improvement of Pseudorange Measurements Accuracy by Using Fast Adaptive Bandwidth Lock Loops, ION GPS 2000 13th International Technical Meeting of the Satellite Division of the Institute of Navigation, Salt Lake City, USA, 2000.

[7] J. B. Zhang, S. F. Zhang, Q. Hu, Y. Jiang, and X. Sun, Design of GNSS Carrier Tracking Loop Base on Adaptive Gain Control, Cross Strait Quad-Reginal Radio Science and Wireless Technology Conference, 2011, Harbin, China, 2011.

[8] J. C. Ding, Z. B. Luo, and L. Zhao, GNSS Carrier Tracking Loop with Loop Gain Control Factor, International Conference about Information and System, 2015, Harbin, China, 2015.

[9] F. Legrand and C. Macabiau, Results of the Implementation of the Fast Adaptive Bandwidth Lock Loops on a Real GPS Receiver in a High Dynamics Context, GNSS 2001,
International Conference on Global Navigation Satellite System, Sevilla, Spain, 2001.

[10] F. Legrand and C. Macabiau, "Real-time minimization of the total tracking error in phase and delay lock loops-a second approach of the fast adaptive bandwidth algorithm," in Proceedings of the 57th Annual Meeting of the Institute of Navigation, Albuquerque, NM, USA, 2001.

[11] K. Borre, D. M. Akos, N. Bertelsen, P. Rinder, and S. H. Jensen, A Software-Defined GPS and Galileo Receiver: A SingleFrequency Approach, Springer Science \& Business Media, 2007.

[12] J. B.-Y. Tsui, Fundamentals of Global Positioning System Receivers: A Software Approach, Jone Wiley \& Sons, 2000.

[13] J.-H. Won, D. Dötterböck, and B. Eissfeller, "Performance comparison of different forms of Kalman filter approaches for a vector-based GNSS signal tracking loop," Navigation, vol. 57, no. 3, pp. 185-199, 2010.

[14] G. Xie, Principles of GPS and Receiver Design, Publishing House of Electronics Industry, Beijing, 2009.

[15] E. D. Kaplan and C. Hegarty, Understanding GPS: Principles and Applications, Artech house, 2005.

[16] F. M. Gardner, Phase Lock Techniques, John Wiley \& Sons, 2005.

[17] C. R. Hamm, W. S. Flenniken, D. M. Bevly, and D. Lawrence, "Comparative performance analysis of aided carrier tracking loop algorithms in high noise/high dynamic environments," in Proceedings of the 2004 ION GNSS Conference, Long Beach, California, USA, 2004.

[18] P. D. Groves, "GPS signal-to-noise measurement in weak signal and high-interference environments," Navigation, vol. 52, no. 2, pp. 83-94, 2005.

[19] P. L. Kazemi, "Optimum digital filters for GNSS tracking loops," in Proceedings of ION GNSS, 2008, Savannah, GA, USA, 2008.

[20] P. A. R. Roncagliolo, C. E. De Blasis, and C. H. Muravchik, "GPS digital tracking loops design for high dynamic launching vehicles," in 2006 IEEE Ninth International Symposium on Spread Spectrum Techniques and Applications, Manaus-Amazon, Brazil, August 2006. 


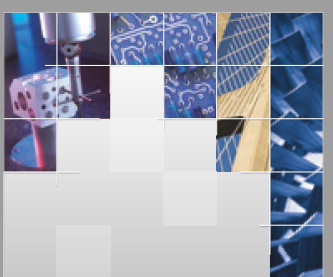

\section{Enfincering}
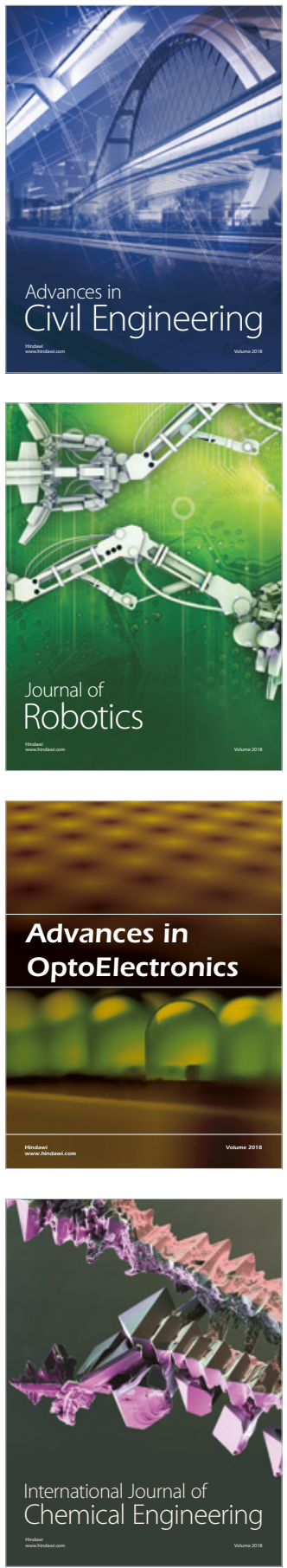

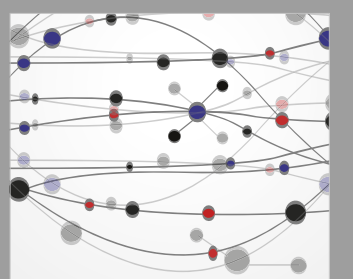

\section{Rotating \\ Machinery}

The Scientific World Journal

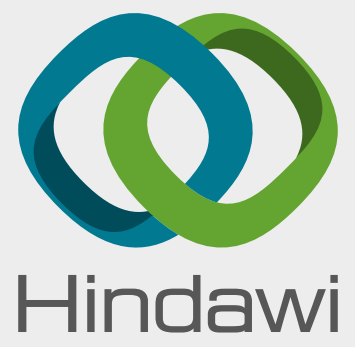

Submit your manuscripts at

www.hindawi.com
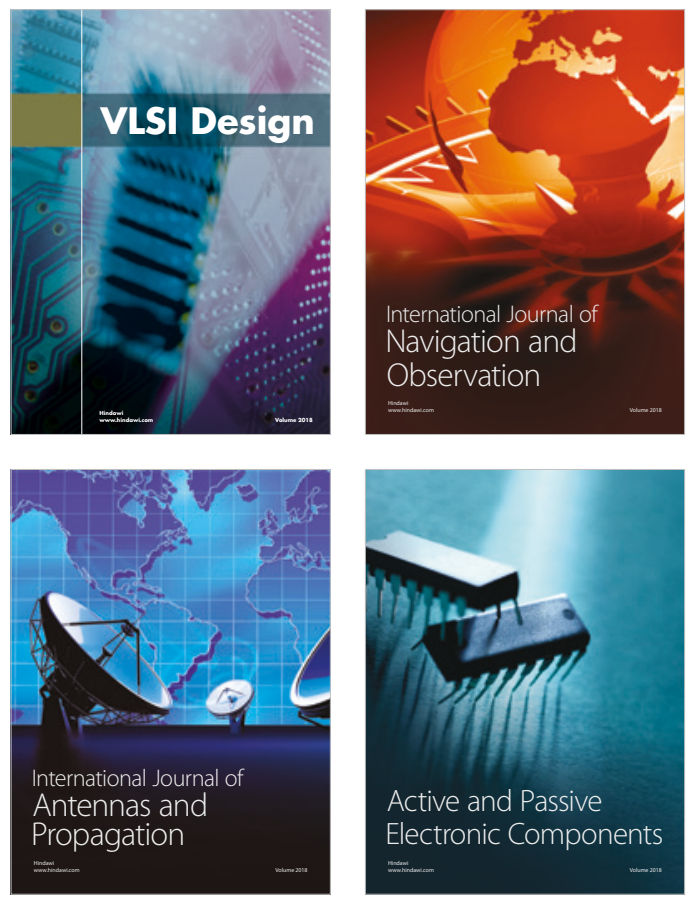
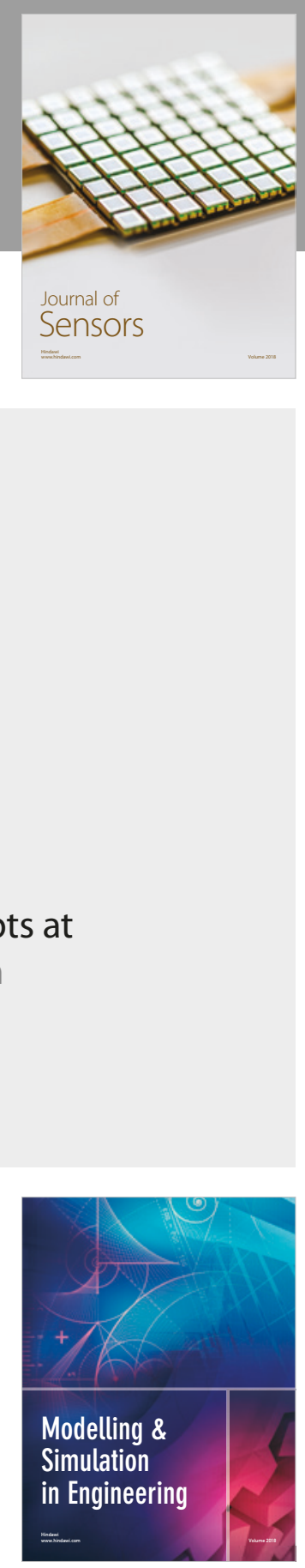

\section{Advances \\ Multimedia}
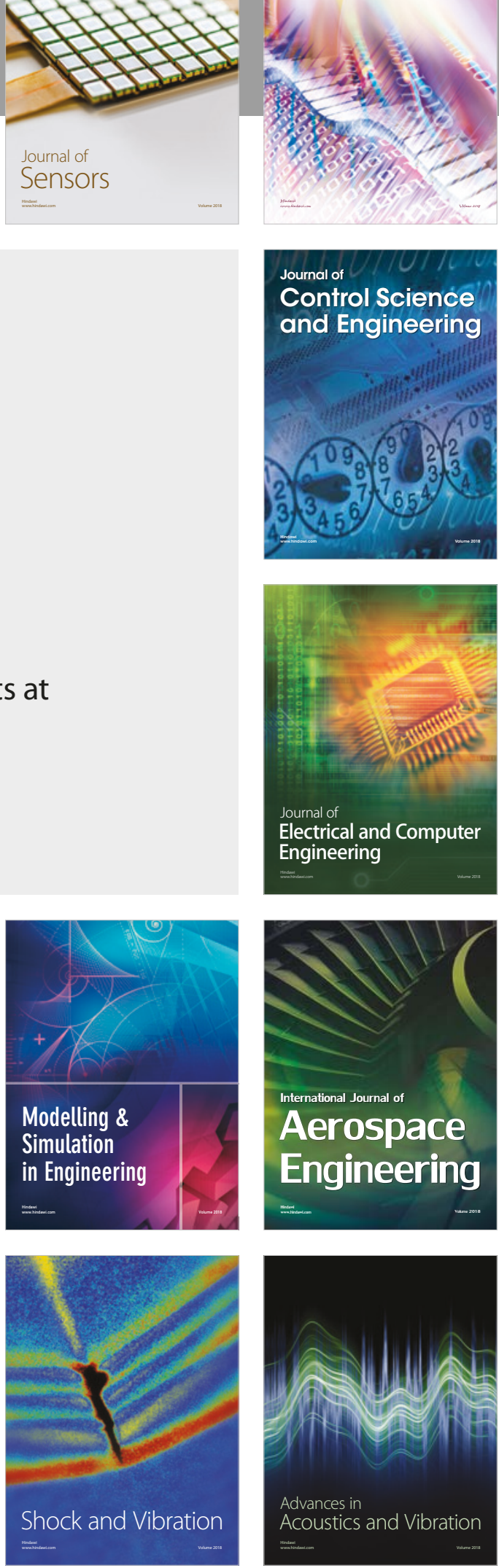